\title{
Proceeding
}

Supplementary Issue: Summer Conferences of Sports Science. Costa Blanca Sports Science Events, 25-26 September 2020. Alicante, Spain.

\section{Effects of two different physical education teaching approaches on the levels of enjoyment in the Italian primary school students}

\author{
ROBERTO COPPOLA ${ }^{1,2} \triangle$, SALVATORE PIGNATO1 ${ }^{1}$, FRANCESCO SGRÒำ, MARIO LIPOMA ${ }^{1}$ \\ ${ }^{1}$ Faculty of Human and Society Sciences, University of Enna "Kore", Enna, Italy \\ ${ }^{2}$ Faculty of Kinesiology, University of Split, Split, Croatia
}

\begin{abstract}
Physical education allows children to develop motor skills, but it has an impact on affective domain too. The aim of this study was to analyse the effects of two different teaching approaches on the levels of enjoyment of 9-year-old pupils who attended the fourth grade of the primary school. A pre- post- repost design was used as a mean of a longitudinal study. The participants involved were divided into two groups: Teaching skill group (TS) and Teaching games group (TGM). The TS group followed a protocol using the traditional skillbased approach, while the TGM group followed the Tactical Game Model approach, highlighting tactical problems and awareness. The level of enjoyment was assessed by administering the PACES questionnaire. The results show no statistically significant difference, but it can be seen that the TGM group had a more positive trend in enjoyment levels than the TS group. Furthermore, carrying out an analysis by gender it was seen that the females of the TGM group had increases in the scores on the positive scale in the post intervention. In this respect, the physical education teacher has to account for affective learning outcomes when they identify the teaching methods for their courses.
\end{abstract}

Keywords: Physical education; Teaching style; Enjoyment; Primary school; Assessment.

Cite this article as:

Coppola, R., Pignato, S., Sgrò, F., \& Lipoma, M. (2020). Effects of two different physical education teaching approaches on the levels of enjoyment in the Italian primary school students. Journal of Human Sport and Exercise, 15(4proc), S1251-S1261. doi:https://doi.org/10.14198/ihse.2020.15.Proc4.26

Corresponding author. Faculty of Human and Society Sciences, University of Enna "Kore", Cittadella Universitaria, 94100, Enna, Italy. https://orcid.org/0000-0001-7888-2208

E-mail: roberto.coppola@unikore.it

Abstract submitted to: Spring Conferences of Sports Science. Costa Blanca Sports Science Events, 19-20 June 2020. Alicante, Spain.

JOURNAL OF HUMAN SPORT \& EXERCISE ISSN 1988-5202

(c) Faculty of Education. University of Alicante

doi:10.14198/jhse.2020.15.Proc4.26 


\section{INTRODUCTION}

The movement, whether carried out within the school system or if carried out in a single daily life, is essential for the well-being of each individual, especially when it is practiced from an early age. The movement represents in fact a tool that facilitates not only the physical development of the child but also and above all the emotional and intellectual development, a development that will make the child aware of himself, of his limits and his abilities.

"The body is the first vehicle by which the individual experiences the environment, it is the first channel of communication between him and others and will continue to be the privileged intermediary in the relationship with others, between the internal and external world" (Federici, Valentini, \& Tonini Cardinali, 2008).

The first place in which the child begins to undertake an autonomous learning path, detached from the control of parental figures, is certainly the school, an institution that must be able to implement a motor path that develops greater autonomy and self-awareness in the child, factors that the child will internalize in order to be able to fit into his community of reference. However, it is difficult for the child to carry all the benefits deriving from physical activity beyond school time. Recent statistics relating to the independent mobility of children in European countries, put Italian children in the fourteenth and last place, together with Portugal, as regards the development of autonomous movement performed daily, with a delay of three - four years compared to the total average European (Borgogni \& Farinella, 2017).

The causes mainly concern the difficulty of finding open and safe spaces equipped with valid supports and capable of containing the child's movement, a deficiency that leads the parent to protect the child by preferring the comfortable use of television and electronic games, rather than seeking alternatives that guarantee an active and participatory growth. The negative effects of smartphone use are showed in a recent work by Greco and colleagues (Greco, Tambolini, Ambruosi, \& Fischetti, 2017). By abandoning the comfort typical of Western living, parents must be able to play a fundamental role in the education of the child, not leaving their child in front of sedentary games that do not develop their imagination and creativity but must help to encourage the enrolment in outdoor sports courses: athletics, swimming, basketball, etc. We need to open a little parenthesis by remembering that by sporting activity, especially in the school environment, we mean the game-sport and not the competitive practice of a sport discipline both for reasons related to the psychomotor area and for reasons related to the problems caused by "trauma" due to high-level sports practice (Notarnicola et al., 2012). What are the culturally valid choices that parents can make to encourage children to approach sports? The choices in this case can be various, choices that must never be made setting aside the individuality of the child but must instead be able to respect their preferences and their character. For example, if we are dealing with a particularly shy child, team sports such as football, basketball or volleyball will help him to grow the importance of the group and of being together. If, on the other hand, we are dealing with a particularly anxious or aggressive child, boxing will help him to discharge in the sport all the energies that negatively condition his character and prevent him from living harmoniously his experiences. An increasing number of researches has focused its attention on the factors that influence adherence to physical activity. Several researchers have suggested that the sensation of pleasure and enjoyment may play an important role in adhering to one exercise or exercise program rather than another (Dishman et al., 2005; Heinzelmann \& Bagley, 1970; Martin \& Dubbert, 1982; Wankel, 1985).

With physical education, the school therefore has the opportunity to intervene in the educational process of everyone, a process that seeks to detach itself from purely intellectualistic notions in order to approach the general growth of the child, both from a physical and mental point of view. In fact, a physical education 
program should be organized considering not only the psychomotor domain of learning but also the affective one. The affective domain of learning is closely connected to the psychomotor one, as especially in younger pupils, the fun experienced during motor-sport activity leads to greater involvement and a lower risk of abandonment in the case of extracurricular sports activities (Ciocan \& Milon, 2017). The educator, for each stage of the child's development, will program for him the widest possible physical base, proposing activities that stimulate the student to more active and integrated participation, awakening that sense of autonomy often hidden by rigid family control. The scientific literature mainly differentiates two teaching styles (i.e. student-centred and teacher-centred). In the Italian school, a teacher-centred approach is mostly used, especially where the activities are not conducted by an expert in physical education and in primary school (where the teacher specialized in physical education is not provided).

Physical education is aimed at a subject who lives the didactic experience through the knowledge he begins to have of his own body, a body that must not be closed in on itself but which is called to interact with others through the rules that are present. within a given environment. This relationship can be defined as a relationship in continuous transformation, capable of involving individual potentials, thus generating the birth of new knowledge and skills (Lipoma, 2014). Without this active and participatory awareness, we cannot speak of physical education, an education that leads the child to gradually build his own identity and to fit into his own reference group through respect and knowledge of others. Another aspect that should be taken care of concerning the discipline of school physical education is that relating to the assessment of skills and competences. recent studies show that through the use of low-cost assessment tools (Sgrò, Licari, Coppola, \& Lipoma, 2015; Sgrò et al., 2015) and standardized motor tests it is possible to make assessments that are not only more precise, but also with greater reliability (Sgrò et al., 2017).

In recent years, efforts have been made to build a motor education that was capable of addressing the possible disabilities of the subjects, both physical and learning, making sure that each individual could manifest their potential without limitations. Everyone is in fact called to express their talent and the school must not shy away from this task but must be the first system capable of supporting the difficulties of each student, in such a way as to express through training the value and cultural depth that every good school must have within it (Ceciliani, 2018).

The Sport Education (SE) is an educational model that links sport in physical education to a wider sporting culture (Siedentop, 1994). Focusing essentially on the notion of authenticity, it is Siedentop's belief that the essential characteristics of the sport that lead to its attractiveness are rarely reproduced in physical education teaching. SE places students in small teams and leads them through a series of skill practices (planned and implemented by teachers and coaches) and through developmentally appropriate games conducted as if they were genuine competition. In contrast to the more common physical education game context where students play in unanswered matches, Sport Education students become members of teams who stay together for the duration of a season and participate in games that are modified in the number of players per team. Therefore, Sport Education is designed to offer students a more complete sports experience than that of a simple isolated player. Games help students develop a sufficient level of skill so that they experience the joy and pleasure that perhaps will provide them with continued motivation to continue playing later in life (Allison, Pissanos \& Turner, 2000; Corbin, 2002; Griffin et al., 1997). Sports pedagogues have focused on the analysis and comparison of teaching-learning processes which are mainly based on "technique" (i.e. motor and sport skills) or on "tactical" aspects (i.e. tactical awareness). Recently, this "gap" that separates technical skills from tactical ones seems to be overcome through the use of Game Centred Approach (GCA) that favour learning contexts allowing not only the full participation and involvement of students (Dyson, Griffin, \& Hastie, 2004) but also managing to link technical skills to that tactical awareness (Mitchell, Oslin, \& 
Griffin, 2013). One of the main tasks of the teacher is to translate the aspects characterizing the discipline into targeted and effective educational actions, planning both the objectives concerning the development of motor skills and the objectives relating to motor learning processes, achievable through a great variety of tasks and interaction between the various teaching styles (Rink, 2002). In physical education, the use of different teaching styles is a necessary prerequisite for the motivation and active participation of students and to promote learning processes according to personalized levels. Mosston elaborated the spectrum of teaching styles by developing the educational relationship between pupil and teacher, in curricular and experimental school contexts (Mosston, 1994). For the teacher, the need to use different teaching styles in the teaching process is related to various factors including the individual characteristics of the students, the diversity of the objectives of physical education involving the cognitive (Russo, Nigro, Raiola, \& Ceciliani, 2019), social and affective area of the person as a function of motor skills and the context that strongly conditions the choice of activities and the use of different organizational methods. Summarizing the teaching styles can be divided into: teacher-centred and student-centred. The aim of this research is to analyse the effects of these two different approaches on the affective domain of learning.

\section{METHOD}

\section{Participants}

This experimental research has as its object the analysis of the effects of different sport-education approaches on the levels of enjoyment of fourth grade children with an average age of 9 years (mean age: $8.6 \pm 0.4$ years old) of an Italian primary school, in a longitudinal study (pre- post-repost design). The sample, composed by 66 pupils, was homogeneously divided into two groups: Teaching Skills group (TS) (teachercentred approach) made up of 32 pupils (17 females and 15 males) and the Tactical Game Model group (TGM) (student-centred) composed of 34 pupils (18 females and 16 males). The protocol lasted 22 weeks of which the first and last were used for pre and post assessment. The repost assessment was carried out after one month. The lessons were conducted to both groups by an expert with a degree in Motor and Sports Sciences, as part of the "Sport di Classe" project (CONI) for one lesson per week (1 hour per lesson). According to a previous study about the recommendations on sampling and selecting participants to guarantee ecological validity in the education research, the different classes have been assigned entirely to one group or the other (Harvey \& Jarrett, 2014). All participants provided informed consent forms signed by their parents or legal guardians. The level of enjoyment was assessed by administering the 16-item PACES (Physical Activity Enjoyment Scale) questionnaire to all participants (Carraro, Young, \& Robazza, 2008; Carraro, 2012), in three stages: pre-intervention, post-intervention and at the beginning of the following school year. The Ethical Committee of the University of Enna "Kore" approved the design and the methodological procedures here used.

\section{Teaching skills and Tactical Game Model' protocols}

As regards the TGM group, the guidelines of Mitchell and colleagues (2013) were followed and for each lesson the following were defined: a tactical problem, a lesson focus and an objective. A multi-sport teaching intervention was carried out and included both invasion and net games (basketball, handball, soccer and volleyball). Each lesson was organized by providing: Game 1 - Practice task - Game 2 - Closure. The first game was used to introduce, through an addictive game, the lesson's tactical problem, whereas the second game was used to reinforce the focus of the lesson. The practice task was focused on the development of movements and skills related to the lesson's tactical problem. Closure was organized as a questioning time used to verify how the students had a focus on the tactical problem of each lesson and which strategies were proposed to solve that problem. The lessons, proposed to the TGM group, were planned following the indications of Mitchell, Oslin \& Griffin (2013), which concern the age group from 7 to 9 years. The "Level 1" 
lessons start with a simplified version. The tactical problems faced are limited to the essential elements of invasion games for beginners such as maintaining possession, penetrating and attacking, starting and restarting the game. As for the net games, the tactical problems were explained in the first lesson in which a part of familiarization with the playing areas (marked with coloured adhesive tape) was also provided. During the planning phase of the protocol, the following were taken into consideration: 1) the modified equipment, in particular the balls (for example: reduced size), allow children to play more effectively and fluently; 2) only a limited number of ideas and suggestions are provided, providing the student with only the essential input to help him in the acquisition of skills, preventing an overload of information; 3 ) the first lesson (for each of the four proposed sports disciplines), at each level of complexity, is dedicated to the organization of the teams, the assignment of fields and the explanation on how to play.

The Teaching Skills group followed a protocol (teacher-centred approach) in which the traditional model of teaching in physical education was implemented, that is, through a skill-based approach, oriented to the development of technical motor skills included in the physical education curriculum in primary school. Each lesson consisted of an introductory activity, a part dedicated to the practice of a technique and a final game to conclude the lesson. As for the sports-games proposed, they were the same as for the TGM group (basketball, handball, soccer and volleyball). During the central part of each lesson, games were proposed using both conventional tools (i.e. balls, frisbees, circles, cones, etc.) and unconventional tools (for example small tools to perfect the throw that the pupils built at home called "Peteke") also focusing on the fundamental skills typical of each of the disciplines. During the final part of three lessons for each of the proposed disciplines, small matches were organized as a Small Sided Games. In the table 1 are reported the lesson focus and the tactical problem related to these two approaches.

Table 1. Lesson focus and tactical problem of each lesson for TS and TGM group respectively.

\begin{tabular}{llll}
\hline Lesson & Game & TS - Lesson focus & TGM - Tactical problem \\
\hline 1 & & & pre assessment \\
2 & Basketball & Passing & Foul shot \\
3 & Basketball & Rebounding & Maintaining possession of the ball \\
4 & Basketball & Dribbling & Attacking the basket \\
5 & Basketball & Shooting & Defending space \\
6 & Basketball & Round-up & Winning the ball \\
7 & Soccer & Passing & Maintaining possession of the ball \\
8 & Soccer & Dribbling & Attacking the goal \\
9 & Soccer & Shooting & Defending space \\
10 & Soccer & Trapping & Winning the ball \\
11 & Soccer & Round-up & Free kick - attacking and defending \\
12 & Handball & Passing & Keeping possession of the ball \\
13 & Handball & Catching & Penetrating the defence and attacking the goal \\
14 & Handball & Throwing & Defending space \\
15 & Handball & Shooting & Winning the ball \\
16 & Handball & Round-up & Restarting from violations \\
17 & Volleyball & Passing & Setting up an attack \\
18 & Volleyball & Spiking & Winning a point \\
19 & Volleyball & Digging & Attacking as a team \\
20 & Volleyball & Serving and Crushing & Defending space on your own court \\
21 & Volleyball & Round-up & Defending against attacks - as a team \\
22 & & & post assessment \\
\hline
\end{tabular}




\section{Statistical analysis}

The statistical analysis was carried out through the use of the SPSS v.21.0 (SPSS Inc. Chicago, IL, USA). After verifying the distribution of data through the analysis of skewness and kurtosis and the KolmogorovSmirnov test, an ANOVA was carried out to analyse the effects of the two approaches in the three administrations of the questionnaire (pre- post- and repost). Then, a post-hoc test was carried out to verify the differences between the results at the three times of administration of the test (pre- post- repost test). Finally, descriptive plots were carried out to graphically verify gender differences.

\section{RESULTS}

The descriptive analysis, the ANOVA and the Bonferroni post-hoc test are reported in the Table 2, 3 and 4, respectively. Finally, an analysis by gender and by group was carried out in the three observation intervals. Regarding the results of the positive scale, while the males of the TGM group did not show variations in the levels of the positive scale of enjoyment, the females, although starting from a lower level, had increases in the scores of the positive scale in the post intervention which means that the protocol used on the latter a positive effect, albeit not statistically significant as highlighted above, which is lost as soon as the project is completed (repost) (Figure 1).

Table 2. Descriptive analysis of the PACES' scales in the two groups.

\begin{tabular}{lllllllll}
\hline & \multicolumn{3}{l}{ Teaching Games group (TG) } & \multicolumn{3}{l}{ Teaching Games group (TG) } \\
\cline { 2 - 9 } Time & Positive Scale & Negative Scale & \multicolumn{2}{l}{ Positive Scale } & \multicolumn{2}{l}{ Negative Scale } \\
\cline { 2 - 9 } & Mean & SD & Mean & SD & Mean & SD & Mean & SD \\
\hline Pre & 4.126 & 0.565 & 1.243 & 0.347 & 4.222 & 0.514 & 1.423 & 0.542 \\
Post & 4.261 & 0.717 & 1.286 & 0.557 & 4.150 & 0.512 & 1.397 & 0.494 \\
Repost & 4.197 & 0.713 & 1.450 & 0.530 & 4.208 & 0.652 & 1.639 & 0.607 \\
\hline
\end{tabular}

Note: Pre: Pre-Training Assessment; Post: Post-Training Assessment; Repost: Repost-Training Assessment. SD: Standard deviation.

Table 3. ANOVA results.

\begin{tabular}{llllll}
\hline Group & PACES' Scale & $\boldsymbol{d}$ & $\mathbf{F}$ & $\boldsymbol{p}$ & $\boldsymbol{\eta}^{\mathbf{2}}$ \\
\hline TG & Positive & 2.000 & 0.345 & .709 & 0.007 \\
TG & Negative & 2.000 & 1.709 & .186 & 0.033 \\
TGM & Positive & 2.000 & 0.148 & .863 & 0.003 \\
TGM & Negative & 2.000 & 1.868 & .160 & 0.039 \\
\hline
\end{tabular}

Note: $T G$ = Teaching Skill group; TGM = Tactical Game group; $d f=$ degrees of freedom; $F=F$ value; $p=p$-value; $\eta^{2}=$ eta squared.

Table 4. Bonferroni post-hoc test result.

\begin{tabular}{lllllll}
\hline \multirow{2}{*}{ TG group } & & & \multicolumn{2}{c}{ Positive Scale } & \multicolumn{2}{l}{ Negative Scale } \\
\cline { 3 - 6 } & & & $\mathbf{t}$ & $\mathbf{p}$ bonf & $\mathbf{t}$ & $\mathbf{p}$ bonf \\
\cline { 3 - 6 } & Pre & Post & -0.830 & NS & 0.358 & NS \\
& & Repost & -0.439 & NS & -1.392 & NS \\
\multirow{2}{*}{ TGM group } & Repost & 0.392 & NS & -1.750 & NS \\
& Pre & Post & 0.513 & NS & -0.191 & NS \\
& & Repost & 0.100 & NS & -1.761 & NS \\
& Post & Repost & -0.413 & NS & -1.570 & NS
\end{tabular}

Note: Pre: Pre-Training Assessment; Post: Post-Training Assessment; Repost: Repost-Training Assessment; $t=t$-score; $p$ bonf $=$ $p$ value; $N S=$ no statistically significant difference. 
a)

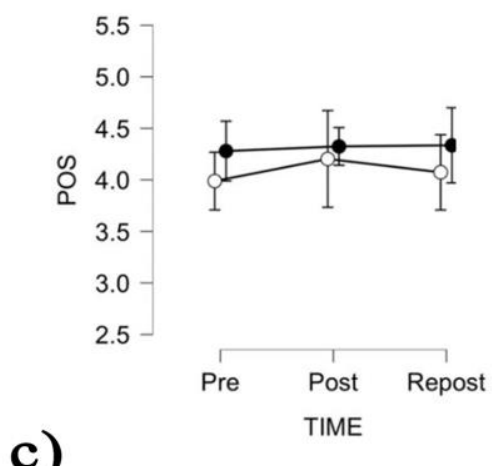

c)

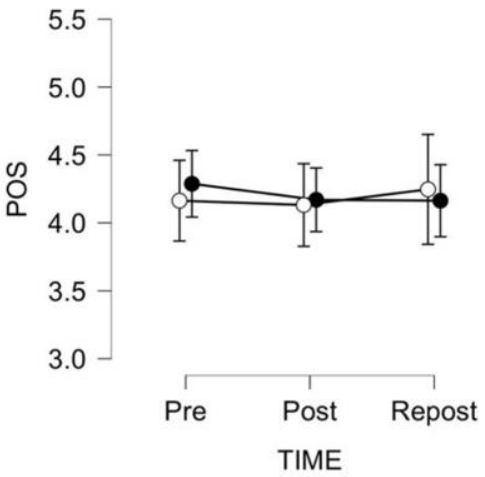

b)

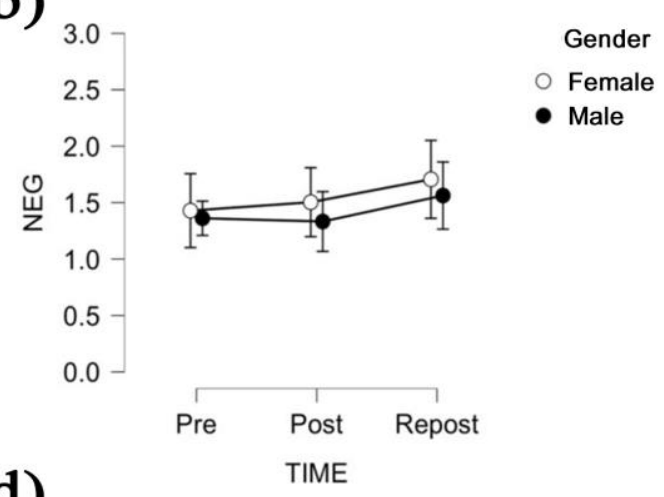

d)

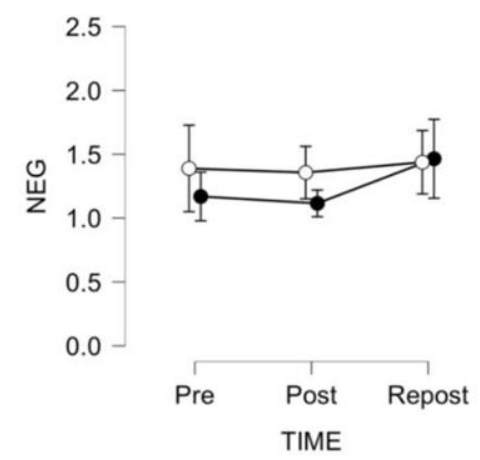

Note: a) descriptive plot related to the positive PACES' scale of the TGM group; b) descriptive plot related to the negative PACES' scale of the TGM group; c) descriptive plot related to the positive PACES' scale of the TS group; d) descriptive plot related to the negative PACES' scale of the TS group; Pre: Pre-Training Assessment; Post: Post-Training Assessment; Repost: Repost-Training Assessment.

Figure 1. Descriptive plots related to the positive and negative scales of the two groups.

\section{DISCUSSION}

The results refer to a relatively small sample, but already provide points for reflection and the basis for further research. In fact, although there were no statistically significant differences between the two groups, it can be said that the Teaching Games group had a more positive trend in enjoyment levels than the Teaching skills group. Regarding the gender differences, although no statistically significant differences were found, through the use of a descriptive plot it is possible to observe a different behaviour. This difference is present for female pupils and the better result of the post treatment seem to fade in the repost test.

The school age is a sensitive phase for the child's development in terms of the development of fundamental motor skills. The school has primary responsibility in promoting activities that reflect the advancements of scientific research, as through educational and cultural renewal it supports the development and enhancement of childhood. The re-evaluation, in recent years, of the body and its enormous potential has given the person a new awareness of their possibilities and has broadened the field in some sectors such as sport, whose elitist practice often gave rise to processes of exclusion. The traditional model of sport, therefore, over time has taken on new and broader characteristics, the motor-sports activities proposed in a playful form are in fact increasingly perceived as a real social need. The gradual transition from purely playful activities to forms of active play, should therefore be configured as a didactic process of kindergarten and primary school, reconsidering corporeality and the activities connected to it as expressions of personality. 
The aim of this research work was to evaluate the effect of two different sport education protocols with two different approaches (i.e. teacher-centred, student-centred) on the levels of enjoyment in primary school children. Although neither of the two protocols used had such an effect as to determine statistically significant differences in the three observation intervals, there is still a positive trend in the Teaching Games group. The non-significance of the results is one of the limitations of this research, this may be the result of a short intervention carried out for only 1 hour a week. The non-statistical significance of the results may also be due to the specialized teacher's approach. In fact, it was the teacher himself who administered both protocols. Despite emerging support for the use of PACES in adolescents, there is little evidence of validity for the use of PACES in children under the age of 12 (Carroll et al., 2001; Carney et al., 2012). Since young children do not possess the same level of understanding or cognitive ability as those who are older, psychological tools designed for adults and adolescents are often not suitable for this population (De Civita et al., 2005). In particular, the 6 to 12 year period is an important developmental time in which children develop complex cognitive skills such as the ability to use personal experiences to make decisions (Stone \& Lemanek, 1990; Tortella \& Fumagalli, 2017) and to rely on mental cues to understand emotions. Here is some research that used PACES with children with good reliability results.

A study by the National Institute of Health sought to determine the reliability and validity of the physical activity enjoyment scale (PACES) in elementary school children in the United States as part of the FitKid Project. The sample consisted of 564 third-grade students (mean age $=8.72 \pm .54$ ) interviewed at the beginning of the fall semester. The enjoyment of physical activity was evaluated by the revised PACES, which was originally designed to measure the positive effects associated with physical activity involvement in college students (Kendzierski \& DeCarlo, 1991). Scores on PACES were significantly correlated with task orientation $(r=.65, p<.01)$, with athletic competence $(r=.23, p<.01)$, and with perception of physical appearance $(r=$ $.20, p<.01)$ each of them evaluated through a specific questionnaire. The results of the present study suggest that PACES has demonstrated promising structural validity and internal consistency as a measure to assess AP-related enjoyment in young European American and African American children. However, despite acceptable indices of internal consistency, relationship sizes for concurrent validity were relatively weak compared to a similar study in 11 - and 13-year-old girls.

The results of the following work are in agreement with those obtained in a previous work (Robertson, 2016). In that study no-statistically significant results were found in relation to PACES after carrying out a TGfU protocol. Enjoyment was identified as an important factor influencing physical activity in two reviews (Health Education Authority, 1997), showing a strong positive relationship across a series of studies. The enjoyment of physical activity has been shown to mediate the effect of a comprehensive school intervention designed to promote physical activity in adolescent girls (Dishman et al., 2005). The evidence gathered to date is however sufficient to encourage the use of enjoyment as a variable in interventions designed to increase physical activity and given the strong link that has now been established, future research should examine the enjoyment as a variable within the school context that is still little investigated.

\section{CONCLUSION}

A recent work has highlighted how the TGM approach is functional both for the personal development of students and for the learning of skills that can go beyond motor-sports practice (curricular and extracurricular), also affecting pleasure. linked to the latter (Sgrò \& Lipoma, 2019). Recent scientific publications show how the level of motor skills in primary school pupils has dropped and how this is a possible consequence of the absence of the specialist in motor science in the Italian primary school (Sgrò, Quinto, Platania, \& Lipoma, 2019). 
Interventions in physical education classes in primary school should be designed both to foster adequate development of basic motor skills and to develop tactical skills, important for individual and team sports. Furthermore, it is essential to be able to create an environment in which children can perceive a motor activity as fun, so as to encourage them more to continue their practice even outside the school context. Furthermore, considering the age range of the following work, it is also essential to consider the duration of the activity in order to have greater results on the affective domain of learning.

It is therefore necessary that structured and oriented interventions, especially those in primary school, must have a sufficient length to have a greater impact on the levels of enjoyment. Teacher training should be reviewed and school organizational issues about PE taught time and facilities should be realistically analysed to highlight the difficulties that teachers encounter and which often prevent the development of the part of the curriculum dedicated to physical education (D'Elia, 2019, 2020). The physical education teacher has to account for affective learning outcomes when they identify the teaching methods for their courses.

Finally, the results underline even more the need for specialized teachers in physical and sports activities within the teaching team of the Italian primary school.

\section{REFERENCES}

Allison, P. C., Pissanos, B. W., \& Turner, A. P. (2000). Preservice physical educators' epistemologies of skillfulness. Journal of Teaching in Physical Education, 19, 141-161. https://doi.org/10.1123/jtpe.19.2.141

Borgogni, A., \& Farinella, R. (2017). Le città attive. Percorsi pubblici nel corpo urbano (Vol. 1569). Franco Angeli.

Cairney J., Kwan M., Velduizen S., Hay J., Bray S. \& Faught B. (2012) Gender, perceived competence and the enjoyment of physical education in children: a longitudinal examination. International Journal of Behavioral Nutrition and Physical Activity volume 9, Article number: 26. https://doi.org/10.1186/1479-5868-9-26

Carraro A., Young M., Robazza C. (2008). A contribution to the validation of the Physical Activity Enjoyment Scale in an Italian sample. Social Behavior and Personality, 36, pp. 911-918. https://doi.org/10.2224/sbp.2008.36.7.911

Carraro, A. (2012). Valutare il piacere nelle attività motorie: il Paces-It. Italian Journal of Educational Research, 259-265.

Carrol B. \& Loumidis J., (2001) Children's perceived competence and enjoyment in physical education and physical activity outside school European Physical Education Review. Volume7(1):2443:015111. https://doi.org/10.1177/1356336X010071005

Ceciliani, A. (2018). Didattica integrata quali-quantitativa, in educazione motoria-sportiva, e benessere in età evolutiva. Formazione \& Insegnamento. Rivista internazionale di Scienze dell'educazione e della formazione, 16(1), 183-194.

Ciocan, D. M., \& Milon, A. (2017). Study Regarding the Motives of Children Participation in Sport Activities. Lumen Proceedings, 1, 159-168. https://doi.org/10.18662/lumproc.rsacvp2017.15

Corbin, C. B. (2002). Physical activity for everyone: What every physical educator should know about promoting lifelong physical activity. Journal of Teaching in Physical Education, 21, 128-144. https://doi.org/10.1123/itpe.21.2.128

D'elia, F. (2019) The core curriculum of university training to teach physical education in Italy, Journal of Physical Education and Sport, 19, pp. 1755-1758. 
De Civita M., Regier D., Alamgir AH., Anis AH., FitzGerald M.J., Marra C.A. (2005). Evaluating HealthRelated Quality-of-Life Studies in Paediatric Populations: Some Conceptual, Methodological and Developmental Considerations and Recent Applications. PharmacoEconomics; 23(7):659-685. https://doi.org/10.2165/00019053-200523070-00003

D'Elia, F. (2020) Teachers' perspectives about contents and learning aim of physical education in Italian primary school, Journal of Human Sport and Exercise, 15 (Proc2), pp. S279-S288. https://doi.org/10.14198/ihse.2020.15.Proc2.19

Dishman K.; Motl W.; Saunders R.; Felton G.; Ward S.; Dowda M.; Pate R. (2005) Enjoyment Mediates Effects of a School-Based Physical-Activity Intervention. Medicine \& Science in Sports \& Exercise: Volume 37 - Issue 3 - 478-487. https://doi.org/10.1249/01.MSS.0000155391.62733.A7

Dyson, B., Griffin, L.L., and P. Hastie. 2004. "Sport education, tactical games, and cooperative learning: Theoretical and pedagogical considerations." Quest, 56(2): 226-240. https://doi.org/10.1080/00336297.2004.10491823

Federici, A., Valentini, M., \& Tonini Cardinali, C. (2008). II corpo educante-Unità di apprendimento di educazione motoria per l'età evolutiva. Aracne.

Greco, G., Tambolini, R., Ambruosi, P., Fischetti, F. (2017). Negative effects of smartphone use on physical and technical performance of young footballers. Journal of Physical Education and Sport, 17 (4), pp. 2495-2501.

Griffin, L.L., Mitchell, S.A., Oslin, J.L. (1997). Teaching sport concepts and skills: A tactical games approach. Champaign, IL: Human Kinetics.

Harvey, S., \& Jarrett, K. (2014). A review of the game-centred approaches to teaching and coaching literature since 2006. Physical Education and Sport Pedagogy, 19(3), 278-300. https://doi.org/10.1080/17408989.2012.754005

Health Education Authority (1997) Young People and Physical Activity: A Literature Review. London: Health Education Authority.

Heinzelmann, F., \& Bagley, R.W. (1970). Response to physical activity programs and their effects on health behavior. Public Health Reports, 85, 905-911. https://doi.org/10.2307/4594000

Kendzierski D. \& DeCarlo KJ. Physical activity enjoyment scale: two validation studies. J Sport Exerc Psychol. 1991;13(1):50-64. https://doi.org/10.1123/jsep.13.1.50

Lipoma, M. (2016). Verso i nuovi significati dell'educazione motoria e fisica. Formazione \& Insegnamento. Rivista internazionale di Scienze dell'educazione e della formazione, 14(1), 7-10.

Martin, J.E., \& Dubbert, P.M. (1982). Exercise applications and promotion in behavioral medicine: Current status and future directions. Journal of Consulting and Clinical Psychology, 50, 1004-1017. https://doi.org/10.1037/0022-006X.50.6.1004

Mitchell, S. A., Oslin, J. L., and L. L. Griffin. (2013). Teaching sport concepts and skills: A tactical games approach for ages 7 to 18. Champaign, IL: Human Kinetics.

Mosston, M., \& Ashworth, S. (2002) Teaching physical education. (5th ed.), Boston: Benjamin Cummings. (United States).

Notarnicola, A., Fischetti, F., Gallone, D., Moretti, L., Pignataro, P., Tafuri, S., Moretti, B. (2012) Overload and neovascularization of shoulder tendons in volleyball players, BMC Research Notes, 5. https://doi.org/10.1186/1756-0500-5-397

Robertson, S. (2016). Investigating the Relationship between Teaching Games for Understanding and High School Physical Education Students' Enjoyment, Self-Efficacy, and Intentions to Enroll.

Russo, G., Nigro, F., Raiola, G., Ceciliani, A. (2019) The role of the extra physical activity on memory storage and psychosocial features, Journal of Human Sport and Exercise, 14 (Proc4), pp. S948S956. https://doi.org/10.14198//hse.2019.14.Proc4.57 
Sgrò, F., \& Lipoma, M. (2019). Riflessioni sull'utilizzo dei Tactical Games Model nella scuola primaria. Formazione \& Insegnamento. Rivista internazionale di Scienze dell'educazione e della formazione, 17(3), 193-206.

Sgrò, F., Licari, D., Coppola, R., \& Lipoma, M. (2015). Assessment of balance abilities in elderly people by means of a clinical test and a low-cost force plate. Kinesiology: International journal of fundamental and applied kinesiology, 47(1), 33-43.

Sgrò, F., Mango, P., Pignato, S., Schembri, R., Licari, D., \& Lipoma, M. (2017). Assessing standing long jump developmental levels using an inertial measurement unit. Perceptual and motor skills, 124(1), 21-38. https://doi.org/10.1177/0031512516682649

Sgrò, F., Nicolosi, S., Schembri, R., Pavone, M., \& Lipoma, M. (2015). Assessing vertical jump developmental levels in childhood using a low-cost motion capture approach. Perceptual and motor skills, 120(2), 642-658. https://doi.org/10.2466/10.PMS.120v12x7

Sgro, F., Quinto, A., Platania, F., \& Lipoma, M. (2019). Assessing the impact of a physical education project based on games approach on the actual motor competence of primary school children. Journal of Physical Education and Sport, 19, 781-786.

Siedentop, D. (1998). "What is sport education and how does it work?." Journal of physical education, recreation \& dance, 69(4): 18-20. https://doi.org/10.1080/07303084.1998.10605528

Stone, WL.; Lemanek, KL. (1990) Developmental issues in children's self-reports. In: Harter, S.; La Greca, A., editors. Through the eyes of a child. Boston: Allyn \& Boston.

Tortella, P., \& Fumagalli, G. (2017). The effect of teaching methodologies in promoting physical and cognitive development in children. Physical Activity and Educational Achievement: Insights from Exercise Neuroscience, 297, 303-316. https://doi.org/10.4324/9781315305790-17

Wankel, L.M. (1985). Personal and situational factors affecting exercise involvement: The importance of enjoyment. Research Quarterly for Exercise and Sport, 56, 275-282. https://doi.org/10.1080/02701367.1985.10605374

\section{(9) $\odot \Theta \Theta$}

This work is licensed under a Attribution-NonCommercial-NoDerivatives 4.0 International (CC BY-NC-ND 4.0). 